\title{
DOES REGRETTING FIRST VAGINAL INTERCOURSE HAVE AN IMPACT ON YOUNG ADULTS' SEXUAL BEHAVIOR?
}

Joan-Carles SURIS, MD, MPH, $\mathrm{PhD}^{1}$

Christina AKRE, $\mathrm{PhD}^{1}$

Caroline JACOT-DESCOMBES, MD ${ }^{2}$

Brigitte LEENERS, $\mathrm{MD}^{3}$

Davide MORSELLI, $\mathrm{PhD}^{4}$

Yara BARRENSE-DIAS, MA ${ }^{1}$

${ }^{1}$ Center for Primary Care and Public Health (Unisanté), University of Lausanne, CH-1010 Lausanne Switzerland

${ }^{2}$ Santé Sexuelle Suisse (Sexual Health Switzerland), CH-1003 Lausanne, Switzerland

${ }^{3}$ Department of Reproductive Endocrinology, University Hospital Zurich, CH8091-Zurich, Switzerland

${ }^{4}$ Swiss Center of Competence in Research LIVES, University of Lausanne, CH-1015

Lausanne, Switzerland

Corresponding author:

Prof. JC Suris

Centre universitaire de médecine générale et santé publique Route de la Corniche 10

1010 Lausanne / Switzerland

Telephone: +41213147375

Fax: +41213147373

Email: joan-carles.suris@unisante.ch

The survey was financed by the Swiss National Science Foundation (grant number 32003B_162538)

Conflict of interest: none to declare 
ABSTRACT

Background. The aims of this research were to determine personal differences depending on the reason for regretting or not first vaginal intercourse and its effects on sexual behavior later on among a representative sample of young adults, and to assess the association between reasons for engaging in first vaginal intercourse and regretting it.

Methods. Data were drawn from the Swiss national survey on youth sexual behaviors conducted in 2017 among young adults (mean age 26 years) living in Switzerland. Out of the 7142 participants, 4793 (51\% females) answered the question Looking back now to the first time you had vaginal intercourse, do you think that... with five possible answers: Should not have done it(6.7\%); Should have waited (7.7\%); Should not have waited (7.4\%); Right time (67.9\%); and I don't know (10.3\%). The five groups were compared on sociodemographic and sex behavior-related variables, analyzed separately by gender.

Results. One third of participants regretted their first experience. In the multivariate analysis, compared to the RightTime group, all other groups were more likely to find their first experience unpleasant and to have done it with a casual partner. Those in the I should not have done it and I should have waited groups were also more likely to have done it because of external pressure, especially among females.

Conclusions. Our results underline the significance to choose the right time and the right partner for first vaginal intercourse and the importance of including partner respect and avoiding external pressure as part of sexual education.

Key words: Young adults; Sexual behavior; First intercourse; Regrets 
51

52

53

54

55

56

57

58

59

60

61

62

63

64

65

66

67

68

69

\section{Introduction}

Reasons for engaging in first vaginal intercourse include being in love(1), being curious $(1,2)$, wanting to lose virginity(2), and external pressure(1). However, literature on how the first time was experienced is scarce, in particular the literature examining the first time leading to subsequent regrets.

A study in New Zealand(2) among a birth cohort at age 21 years found that $16 \%$ of men and $54 \%$ of women indicated that they should have waited longer while $11 \%$ and $1 \%$ of them, respectively, thought that they should not have waited that long. In a study carried out in Scotland(3) among close to eight thousand schoolchildren with a mean age of 14 years, $32 \%$ of girls and $27 \%$ of boys having experienced sexual intercourse reported that it happened too early and $15 \%$ and $5 \%$, respectively, that it should not have happened at all. A slightly different approach using a convenience sample $(\mathrm{N}=248)$ of college students(4) found that three quarters of them had regretted their decision to engage in sexual activity. Longitudinal data of British youth aged 13-16 years indicated that $20 \%$ of males and $38 \%$ of females regretted their first time(5). A school sample of 14-18 year-olds in three developing countries(1) found regret rates ranging from $25 \%$ to $32 \%$ for females and from $13 \%$ to $21 \%$ among males. The most recently published study(6) concluded that, among those having had sexual intercourse, one 15 year-old in five had negative feelings about the timing of their first intercourse, with a clear female predominance.

Regretting first sexual intercourse has been associated with female gender(5, 6), not doing it with a steady partner(2, 3, 5), being intoxicated(1, 3-5), being pressured(1, 3-5) or forced(2) by partner, having an older partner(7), not using a condom $(4,7)$, and early age at first intercourse $(5,8)$. 
However, research on regrets about first vaginal intercourse is scarce regarding its impact on future sexual behavior and limited by the methodology used. Most of the studies are schoolbased $(1,3,5,6)$ and, although they include large samples, only a minority of respondents are sexually experienced. Additionally, most of them, due to the samples' age ranges, have also had an early sexual debut, which is associated to regrets later on(5). Moreover, the paper including college students(4) is based on a convenience sample and not generalizable. Finally, the birth cohort study from New Zealand(2) is the one that gives a better temporal perspective as respondents have been sexually active, on average, for four years, but data were collected 25 years ago.

To our knowledge, there is no recent investigation looking at regretting first vaginal intercourse with enough temporal perspective based on a large sample of young adults. The primary aim of this research was to determine personal differences depending on the reason for regretting or not first vaginal intercourse and its effects on sexual behavior in the long run among a nationally representative sample of young adults. Secondarily, we aimed to assess the association between different reasons for engaging in first vaginal intercourse and regretting it.

\section{METHODS}

Data were drawn from the Swiss national survey on youth sexual behaviors, a cross-sectional study conducted in 2017 among a representative sample of young adults (mean age 26 years) living in Switzerland. A complete description of the study can be found elsewhere(9). Briefly, data were obtained from a self-administrated questionnaire on sexual behaviors among young adults. The initial sample was provided by the Swiss Federal Office of Statistics and was representative of the 24-28 years old population living in Switzerland in terms of gender, language (French, Italian or German) and canton of residence. All those included in the initial sample were contacted by postal mail to participate in the study and received an information 
letter together with a unique login code to participate in an online survey. The final sample included 7142 participants (response rate $15.1 \%$, mean age 26.3 years). To correct a slightly over-representation of females from the French part of Switzerland, analyses were weighted by gender and canton of residence. The survey collected sociodemographic and sexual health and behavior data using a life history calendar (LHC) approach. The LHC is a highly structured but flexible approach to data collection that facilitates recall of past events by using the individual's own past experiences as cues for remembering. These cues provide context for retrieval of autobiographical memory and increment the precision of reports(10, 11). The Ethics committee in research of the canton of Vaud gave ethic clearance in agreement with the Swiss law.

Out of the 7142 participants, 4793 (51\% females) answered the question Looking back now to the first time you had vaginal intercourse, do you think that... with five possible answers: I should not have done it $(\mathrm{N}=320,6.7 \%)$; I should have waited longer $(\mathrm{N}=369,7.7 \%)$; I should have done it earlier ( $\mathrm{N}=353,7.4 \%)$; It was about the right time (RightTime; $\mathrm{N}=3255,67,9 \%$ ); and I don't know $(\mathrm{N}=496,10.3 \%)$.

The five groups were compared on age, family socioeconomic status (SES) and perceived pubertal timing. SES was measured though the question Compared to other families in Switzerland, your family financial situation when you were 15 was.... with 7 possible answers ranging from well-below to well-above average and dichotomized into below average and average or above(12). Perceived pubertal timing was assessed through If you think of the age you started puberty, compared to your peers would you say that you were ... with 3 possible answers: early, on time, late (13).

The context of their first vaginal intercourse was analyzed through their debut age (under 16 years / 16 and over)(14,15), whether the experience had been pleasant (yes/no), the type of partner (casual or steady), the partner's age (younger, about the same age, older), condom use 
(yes/no), whether it was also the first time for their partner (yes/no) and the reason for first intercourse.

Nine reasons for first vaginal intercourse were proposed: I was in love, I was curious, I wanted to do like my peers, I was forced, I was intoxicated, I wanted to lose my virginity, I did it without wanting, Other, I can't remember. Due to small group numbers, doing like my peers $(\mathrm{N}=66)$, being forced $(\mathrm{N}=35)$ and doing it without wanting $(\mathrm{N}=57)$ were combined into External pressure. In the same line, I cannot remember $(\mathrm{N}=82)$ and Other $(\mathrm{N}=85)$ were combined into Other. All other options remained as described.

Looking for a possible impact of their first sexual intercourse on their future sexual behavior, we also analyzed aspects of their current sexual life. Studied variables included: number of lifetime sexual partner(s) (one/two-three/four or more), having had a sexual partner in the last 12 months (yes/no), whether they had only had heterosexual relationships (yes/no), being currently with a steady partner (yes/no), satisfaction with their current steady partner relationship (yes/no) and, if they did not have a steady partner at the moment, satisfaction with their last steady partner relationship (yes/no).

In a first step, we completed a bivariate analysis to compare the five groups using chi-square tests for categorical variables and ANOVA for continuous ones. Subsequently we performed a multinomial logistic regression analysis including all significant variables at the bivariate level ( $\mathrm{p}<.05)$ (except reason for first intercourse) using the RightTime group as the reference category. Finally, we carried out a second multinomial logistic regression analysis separately for each reason for first sexual intercourse controlling for variables significant at the bivariate level, using the same reference category. 
146 All analyses were done using Stata 14 (StataCorp, College Station, Texas) and performed

147 separately by gender as males and females do not report the same experiences of their first 148 vaginal intercourse $(1,5,6)$

\section{RESULTS}

Overall, about two-thirds of youths indicated that their first vaginal intercourse happened at the right time, with similar rates for both genders (females: 67.2\%, Males: 68.7\%). Among females, I should have waited was the second most often cited reaction (10.9\%) followed by $I$ don't know (9.6\%), I should not have done it (9.3\%), and I should have done it earlier (3.0\%). Among males, I should have done it earlier (11.9\%) ranked second followed by I don't know (11.1\%), I should have waited (4.3\%) and I should not have done it (4.0\%).

The main reason for first vaginal intercourse was being in love for both females $(56.1 \%)$ and males (42.9\%), followed by I was curious (27.7\% and 38.8\%, respectively) and I wanted to lose my virginity (5.5\% and 9.6\%, respectively). For females, external pressure (4.9\%) ranked fourth, followed by other (3.4\%) and being intoxicated $(2.4 \%)$, while among males, it was other (3.6\%) followed by being intoxicated (3.5\%) and external pressure (1.6\%).

\section{FEMALES}

At the bivariate level, all variables were significant except not having a sexual partner in the last 12 months, currently being with a steady partner, and satisfaction with current or last steady partner (Table 1).

In the multivariate analysis (Table 2), compared to the RightTime group, all other groups were significantly more likely to report that their first experience had been unpleasant and with a casual partner and less likely to have had only one sexual partner in their lifetime.

Those in the I should not have done it group were more likely to have had their first experience before the age of 16 , to report a lower SES and to not having used a condom. 
Those in the I should have waited group were also more likely to be under 16 at their first experience, not to be the first time for their partner and to report a perceived advanced puberty compared to their peers. Those in the I should have done it earlier group were less likely to have engaged only in heterosexual relationships, to have had their first experience before the age of 16, and to have had four or more lifetime sexual partners. Finally, those in the I don't know group were more likely to be in no relationship currently.

Compared to those in the RightTime group, all other groups reported a lower likelihood of being in love as a reason for their first intercourse. The I should not have done it, I should have waited, and I don't know groups were more likely to report external pressure and other reasons, while those in the I should have done it earlier group reported wanting to lose their virginity. The I should not have done it group was less likely and the I should have waited group more likely to report curiosity. Finally, both the I should not have done it and I don't know groups were more likely to indicate that they were intoxicated as the reason for their first vaginal intercourse (Table 2).

\section{MALES}

At the bivariate level all variables but age, SES, currently being with a steady partner and satisfaction with current or last steady partner were significant (Table 3).

Similar to females, the multivariate analysis (Table 4) showed that, compared to the RightTime group, all other groups were significantly more likely to have found their first experience unpleasant and to have had it with a casual partner. The I should not have done it group reported higher odds that their first intercourse was before age 16, that it was not the first time for their partner, and that they had not used a condom. Additionally, they were significantly less likely to have had four or more lifetime sexual partners. The I should have done it earlier group reported being older and less likely to have initiated intercourse before 
age 16. They reported higher odds of their first partner being older, of not being the first time for their partner and of not being currently in a relationship. The I don't know group was less likely to state only one lifetime sexual partner.

All groups but I should have done it earlier were less likely to report being in love and more likely to state external pressure as the reason for their first experience compared to the RightTime one. Those in the I should have done it earlier group were twice as likely to report wanting to lose their virginity as the reason. Finally, those in the I don't know group also reported being intoxicated and other as the reasons for their first vaginal intercourse (Table 4).

\section{DISCUSSION}

The majority of participants indicated that they had their first vaginal intercourse at the right time, as described in the literature $(5,6)$, although results in other school-aged samples showed lower rates(3), probably due to the younger age of the sample's participants. However, gender differences appear for regret reasons: while about ten percent of females report that they should have waited or that they should not have done it, $12 \%$ of males indicate that they should have done it earlier. These results are similar to those found in New Zealand(2). Other studies among samples of adolescents indicated that only one fifth regretted their first experience, but the vast majority in that sample were not sexually initiated(1,6).

The most cited reason for first vaginal intercourse by both genders is being in love, as described by other authors(1), even though others indicate curiosity(2). It is significant to mention that even if those who report external pressure are a small minority, they are more frequent among females. Although the external pressure rates found in our study are much lower than those described among school-aged adolescents $(1,3,5)$, it is worth noting the 
important association for both genders (but much more marked among females) among those regretting having had their first experience too early or thinking that they should not have had their sexual debut at that moment. The lower rate of external pressure found in our study compared to school-based studies can be explained by their young debut age that is linked to regretting later(5). This finding highlights the importance of prevention among young people with a special emphasis on teaching respect for partners together with how to avoid pressure. Although these topics are part of school-based sexual education, this is limited to mandatory education in Switzerland (at ages 13-15). It would be important to add sexual education to review these issues in post-mandatory education as this is when most youths have their sexual debut.

Compared to those indicating that they had their first vaginal intercourse at the right time, all other groups had higher odds of reporting this first experience as unpleasant independently of gender. The fact that their first time was more likely to have happened with a casual partner could explain it, as it has been described that being in a committed relationship increased psychological satisfaction, especially among women(16). Moreover, the often high expectations put on this event may not be reached and be a cause for subsequent regret. Finally, the unpleasantness of this first experience may be linked to their subsequent regret(8) while a more positive first experience has been associated with greater feelings of sexual satisfaction in their current interactions(17).

Among both genders, reporting that they should not have done it is linked to having had vaginal intercourse before age 16 , and to not having used a condom, even though, in line with other studies (5), the vast majority of young adults in our sample had used a condom in their first intercourse and even showed higher rates than others(3). A Scandinavian study reported that the risk of contraceptive non use increased with earlier age at sexual debut among women(18).With the exception of the I should have done it earlier group among males, and 
contrary to other studies $(5,6,8)$, we found no age difference with their first partner. In a similar way, only females in the I should have done it earlier group and males in the I should not have done it one were less likely to have had four or more lifetime partners, while other studies found no difference(5).

Our results show that females in the I should not have done it group reported a lower SES. Moreau et al. (6) described that girls from less affluent families were more likely to report negative feelings regarding their first experience. This finding could also be explained by the fact that a lower SES is often associated to low parental education, which has been correlated to early sexual debut in the literature $(19,20)$.

Females in the I should have done it earlier group were the only ones to show a lower likelihood to have had only heterosexual relationships. Dewaele et al.(21) found similar results among men having sex with men, but we could not find comparable results for women in the literature. Whether this result may be explained by the time needed to assume their sexual preferences remains to be studied among young women. Both females and males in the I should have done it earlier group also were less likely to have had their first experience before age 16. Moreover, females had lower odds of having had four or more lifetime sexual partners, and males to be currently in a relationship. These results are in concordance with the literature indicating that late age at first sex was associated with lower odds of cohabitation and fewer romantic partners in young adulthood(22).

Finally, in our sample, regretting their first vaginal intercourse seems to have little effect on sexual behavior in young adulthood, with the vast majority of participants being currently with a steady partner and satisfied with the relationship they have with her/him even though the initial context was unfavorable. Our findings are in disagreement with the literature. To date, most research on the effects of sexual debut on early adulthood sexual behavior seems to be limited to early sexual intercourse (23) and to be associated to negative consequences 
such as increased number of partners(24-27), sexual intercourse while intoxicated(25), and sexually transmitted infections $(24,26)$. However, there is also evidence indicating that it is rather the context of sexual debut and not necessarily the age which is important, and that experiences happening in negative contexts yield adverse consequences later on (28). Our data do not allow analyzing whether regret was limited to a single first episode or continued over time and we do not know either the sexual trajectories of participants from adolescence into young adulthood. Future research should look at other potential factors influencing young adulthood sexual satisfaction that may buffer a negative initial encounter.

The strengths of this research are that it is based on a nationally representative sample, participants have a sufficient temporal perspective and uses the life history calendar as a method to help participants situate different events in their life. Nonetheless, some limitations need to be discussed. First, we had a low response rate. We were conscious that a sensitive subject such as sexuality and the way we had to contact potential participants (postal instead of electronic mail) could have an important impact on the response rate. For this reason, we decided to start with a very large sample (over 40’000) so that the final sample would be large enough. Second, participants had to answer about events that happened about ten years earlier and a memory bias cannot be excluded. However, using the LHC should minimize it. In the same line, a social desirability bias cannot be disregarded either. However, there is evidence indicating that the LHC method is more prone to open and honest responses(29). Third, participants could only choose one reason for engaging in first vaginal intercourse and motivations could be more complex than that. Fourth, the category external pressure is relatively large and could have different meanings depending on the respondent. Finally, our survey being cross-sectional, causality cannot be determined.

About one third of young adults regret the way they experienced their first vaginal intercourse. Among them, it was much more frequent to experience this event as unpleasant, 
probably because it was more often with a non-steady partner. Although the overall

294 percentage of those regretting does not differ by gender, females seem to have worse

295 experiences than males, especially regarding external pressure. Nevertheless, although this

296 first regretful experience does not seem to have a clear negative impact on their sexual life as

297 young adults, further research needs to be carried out to better define its potential impact. Our

298 results underline the significance to choose the right time and the right partner for the first

299 vaginal intercourse and the importance of including partner respect and teaching skills to

300 avoid external pressure (especially non-consensual sex) as part of school-based sexual

301 education 
303 Compliance with Ethical Standards

304 Conflict of interest. None to declare.

305 Ethical Approval. All procedures performed in studies involving human participants were in

306 accordance with the ethical standards of the institutional and/or national research committee

307 and with the 1964 Declaration of Helsinki and its later amendments or comparable ethical

308 standards.

309 Informed Consent. Informed consent was obtained from all individual participants included 310 in the study. 
1. Osorio A, Lopez-del Burgo C, Carlos S, Ruiz-Canela M, Delgado M, de Irala J. First sexual intercourse and subsequent regret in three developing countries. J Adolesc Health. 2012;50(3):271-8. 2. Dickson N, Paul C, Herbison P, Silva P. First sexual intercourse: age, coercion, and later regrets reported by a birth cohort. BMJ. 1998;316(7124):29-33.

3. Wight D, Henderson M, Raab G, Abraham C, Buston K, Scott S, et al. Extent of regretted sexual intercourse among young teenagers in Scotland: a cross sectional survey. BMJ. 2000;320(7244):1243-4.

4. Oswalt SB, Cameron KA, Koob JJ. Sexual regret in college students. Arch Sex Behav. 2005;34(6):663-9.

5. Wight D, Parkes A, Strange V, Allen E, Bonell C, Henderson M. The quality of young people's heterosexual relationships: a longitudinal analysis of characteristics shaping subjective experience. Perspect Sex Reprod Health. 2008;40(4):226-37.

6. Moreau N, Kolto A, Young H, Maillochon F, Godeau E. Negative feelings about the timing of first sexual intercourse: findings from the Health Behaviour in School-aged Children study. Int J Public Health. 2018.

7. Mercer $\mathrm{CH}$, Wellings K, Macdowall W, Copas AJ, McManus S, Erens B, et al. First sexual partnerships--age differences and their significance: empirical evidence from the 2000 British National Survey of Sexual Attitudes and Lifestyles ('Natsal 2000'). J Adolesc Health. 2006;39(1):87-95.

8. Reissing ED, Andruff HL, Wentland JJ. Looking back: the experience of first sexual intercourse and current sexual adjustment in young heterosexual adults. J Sex Res. 2012;49(1):2735.

9. Barrense-Dias Y, Akre C, Berchtold A, Leeners B, Morselli D, Suris JC. Sexual health and behavior of young people in Switzerland. Lausanne: IUMSP; 2018.

10. Martyn KK, Saftner MA, Darling-Fisher CS, Schell MC. Sexual risk assessment using event history calendars with male and female adolescents. J Pediatr Health Care. 2013;27(6):460-9.

11. Morselli D, Berchtold A, Suris JC, Berchtold A. On-Line Life History Calendar and Sensitive Topics: A Pilot Study. Computers in Human Behavior. 2016;58:141-9.

12. Hibell B, Andersson B, Bjarnason T, Ahlström S, Balakireva O, Kokkevi A, et al. ESPAD Report 2003. Alcohol and other drug use among students in 35 European countries. Stockholm: The Swedish Council for Information on Alcohol and Other Drugs (CAN); 2004.

13. Berg-Kelly K, Erdes L. Self-assessment of sexual maturity by mid-adolescents based on a global question. Acta Paediatr. 1997;86(1):10-7.

14. Baumann P, Belanger RE, Akre C, Suris JC. Increased risks of early sexual initiators: time makes a difference. Sex Health. 2011;8(3):431-5.

15. Wellings K, Nanchahal K, Macdowall W, McManus S, Erens B, Mercer CH, et al. Sexual behaviour in Britain: early heterosexual experience. The Lancet. 2001;358:1843-50.

16. Higgins JA, Trussell J, Moore NB, Davidson JK. Virginity lost, satisfaction gained? Physiological and psychological sexual satisfaction at heterosexual debut. J Sex Res. 2010;47(4):384-94.

17. Smith CV, Shaffer MJ. Gone but not forgotten: virginity loss and current sexual satisfaction. J Sex Marital Ther. 2013;39(2):96-111.

18. Guleria S, Juul KE, Munk C, Hansen BT, Arnheim-Dahlstrom L, Liaw KL, et al. Contraceptive non-use and emergency contraceptive use at first sexual intercourse among nearly 12000 Scandinavian women. Acta Obstet Gynecol Scand. 2017;96(3):286-94.

19. Price MN, Hyde JS. When two isn't better than one: predictors of early sexual activity in adolescence using a cumulative risk model. J Youth Adolesc. 2009;38(8):1059-71.

20. Asamoah BO, Agardh A. Individual- and Family-Level Determinants of Risky Sexual Behavior Among Swedish- and Foreign-Born Young Adults 18-30 Years of Age, Residing in Skane, Sweden. Arch Sex Behav. 2018;47(2):517-28.

21. Dewaele A, Van Houtte M, Symons K, Buysse A. Exploring First Sexual Intercourse, Sexual Orientation, and Sexual Health in Men. J Homosex. 2017;64(13):1832-49.

22. Harden KP. True love waits? A sibling-comparison study of age at first sexual intercourse and romantic relationships in young adulthood. Psychol Sci. 2012;23(11):1324-36.

23. Heywood W, Patrick K, Smith AM, Pitts MK. Associations between early first sexual intercourse and later sexual and reproductive outcomes: a systematic review of population-based data. Arch Sex Behav. 2015;44(3):531-69. 
24. Rissel CE, Richters J, Grulich AE, de Visser RO, Smith AM. Sex in Australia: first experiences of vaginal intercourse and oral sex among a representative sample of adults. Aust N Z J Public Health. 2003;27(2):131-7. 25. Sandfort TG, Orr M, Hirsch JS, Santelli J. Long-term health correlates of timing of sexual debut: results from a national US study. Am J Public Health. 2008;98(1):155-61.

26. Olesen TB, Jensen KE, Nygard M, Tryggvadottir L, Sparen P, Hansen BT, et al. Young age at first intercourse and risk-taking behaviours--a study of nearly 65000 women in four Nordic countries. Eur J Public Health. 2012;22(2):220-4.

27. Prendergast LE, Toumbourou JW, McMorris BJ, Catalano RF. Outcomes of Early Adolescent Sexual Behavior in Australia: Longitudinal Findings in Young Adulthood. J Adolesc Health. 2019;64(4):516-22.

28. Else-Quest NM, Hyde JS, Delamater JD. Context counts: long-term sequelae of premarital intercourse or abstinence. J Sex Res. 2005;42(2):102-12.

29. Fisher CM. Queering Data Collection: Using the Life History Calendar Method With SexualMinority Youth. Journal of Social Service Research. 2013;39(3):306-21.

385 